predicted response, rather than randomisation. Bias can then be minimised by propensity score matching ${ }^{5}$ (controlling for unmeasured bias between study groups), although this method was not employed by Kessing et al.

1 Kessing LV, Hellmund G, Geddes JR, Goodwin GM, Andersen PK. Valproate $v$ lithium in the treatment of bipolar disorder in clinical practice: observational nationwide register-based cohort study. Br J Psychiatry 2011; 199: 57-63.

2 Geddes JR, Goodwin GM, Rendell J, Azorin JM, Cipriani A, Ostacher MJ, et al. Lithium plus valproate combination therapy versus monotherapy for relapse prevention in bipolar I disorder (BALANCE): a randomised open-label trial. Lancet 2010: 375: 385-95.

3 Black $\mathrm{N}$. Why we need observational studies to evaluate the effectiveness of health care. BMJ 1996; 312: 1215-8.

4 Alda M, O'Donovan C. A much needed BALANCE. Bipolar Disord 2010; 12 : 678-80.

5 Rosenbaum PR, Rubin DB. The central role of the propensity score in observational studies for causal effects. Biometrika 1983: 70: 41-55.

Joseph F. Hayes, Unit of Mental Health Sciences, University College London, Charles Bell House, 67-73 Riding House Street, London W1W 7EJ. Email: joseph.hayes@medsch.ucl.ac.uk; David Osborn, Unit of Mental Health Sciences, University College London

doi: 10.1192/bjp.199.4.341b

Authors' reply: We certainly agree on the mentioned advantages and disadvantages of observational studies and on the strengths of combining findings from randomised trials with those of observational studies.

Further, we agree on the possibility of the suggested analyses with 'switch to' and 'add on' as two separate outcomes. We chose the combined outcome measure as using two separate outcome measures (in addition to hospitalisation as an outcome measure) would decrease the statistical power to a low level in some of the analyses. In addition, one of the advantages of using the combined outcome measure is that the results may turn out to be more clear to guide clinical decisions on whether to use lithium or valproate in long-term treatment of bipolar disorder following a number of clinical situations (depression, mania, mixed episode or remission).

Propensity score matching (or other ways of introducing propensity score in the analysis ${ }^{1}$ ) is a viable alternative to the approach based on multiple Cox regression models used in our paper. However, much experience (e.g. Sturmer et $a^{2}$ ) suggests that the results thus obtained would not tend to be substantially different. The limiting factor seems to be the available amount of covariate information.

1 D'Agostino Jr RB. Tutorial in biostatistics. Propensity score methods for bias reduction in the comparison of a treatment to a non-randomized control group. Stat Med 1998; 17: 2265-81.

2 Sturmer T, Joshi M, Glynn RJ, Avorn J, Rothman KJ, Schneeweiss S. A review of the application of propensity score methods yielded increasing use, advantages in specific settings, but not substantially different estimates compared with conventional multivariable methods. J Clin Epidemiol 2006; 59: 437-47.

Lars Vedel Kessing, Psychiatric Center Copenhagen, University Hospital of Copenhagen, Rigshospitalet, Denmark. Email: lars.vedel.kessing@regionh.dk. Per Kragh Andersen, Department of Biostatistics, University of Copenhagen Copenhagen, Denmark

doi: 10.1192/bjp.199.4.342

\section{Role of postcards in reducing suicidal behaviour}

The article by Hassanian-Moghaddam et al provides useful insights into the potential utility of postcard intervention in reducing suicidal behaviour. The authors by virtue of this study have found that among participants who had self-poisoned, nine postcards sent sequentially over a period of 12 months produced reduction in suicidal ideation and suicide attempts. The study deserves accolades for various reasons, including a large sample from a non-Western population and a randomised control design, ensuring an over $90 \%$ retention rate and nearly equal rates of loss to follow-up in both groups. The results of the study are illuminating but their generalisability and applicability in day-to-day clinical practice needs to be analysed against the backdrop of following limitations.

(a) The study provided for assessment of outcomes only at 12 months. It would have been better if the assessments were performed more frequently such as once in 2 or 3 months.

(b) The study at no point assessed suicidal intent among participants.

(c) Instead of employing any standard sampling technique, the participants of the study included consecutive individuals with poisoning, admitted from March to June 2006 in the Loghman-Hakim Poison Hospital.

(d) Baseline assessment did not include a comprehensive psychiatric evaluation that could have ascertained the specific psychiatric diagnosis of the participants and permitted subgrouping of the participants based on psychiatric diagnosis, thereby providing a valuable opportunity to study the differential impact of postcard intervention in reducing suicidal ideation and suicidal attempt among the participants with different psychiatric disorders.

(e) There is no mention in the article of whether the delivery of the postcards was confirmed by the recipients.

(f) The participants were masked to study outcomes but the research psychologist was not masked to allocation, and this could have inadvertently influenced responses at follow-up.

(g) Individuals may have got some clue about the study outcomes from the questions asked of them and this could have influenced the final results of the study.

(h) A small minority of participants withdrew from the postcard intervention but the specific reasons for the same were not assessed.

To make the postcard intervention more acceptable and effective, one needs to ascertain the specific reasons which made the participants withdraw from this intervention.

1 Hassanian-Moghaddam H, Sarjami S, Kolahi A, Carter GL. Postcards in Persia: randomised controlled trial to reduce suicidal behaviours 12 months after hospital-treated self-poisoning. Br J Psychiatry 2011; 198: 309-16.

Anurag Jhanjee, University College of Medical Sciences and Guru Teg Bahadu Hospital, Dilshad Garden, Delhi, India. Email: Anurag_99@hotmail.com; Manjeet Singh Bhatia, University College of Medical Sciences and Guru Teg Bahadur Hospital, Dilshad Garden, Delhi, India

doi: 10.1192/bjp.199.4.342a

Authors' reply: Drs Jhangee \& Bhatia have mentioned a number of strengths and limitations, which were specifically addressed in the paper. The other issues that were raised are addressed below.

(a) Postcards are a minimal intervention sustained over 12 months. Optimal assessment is end of treatment and at follow-up, which allows comparison with similar studies. ${ }^{1,2}$ Repeated contact and assessment might 'wash out' the effect 